\title{
Functional degradation ignites the inflammasome
}
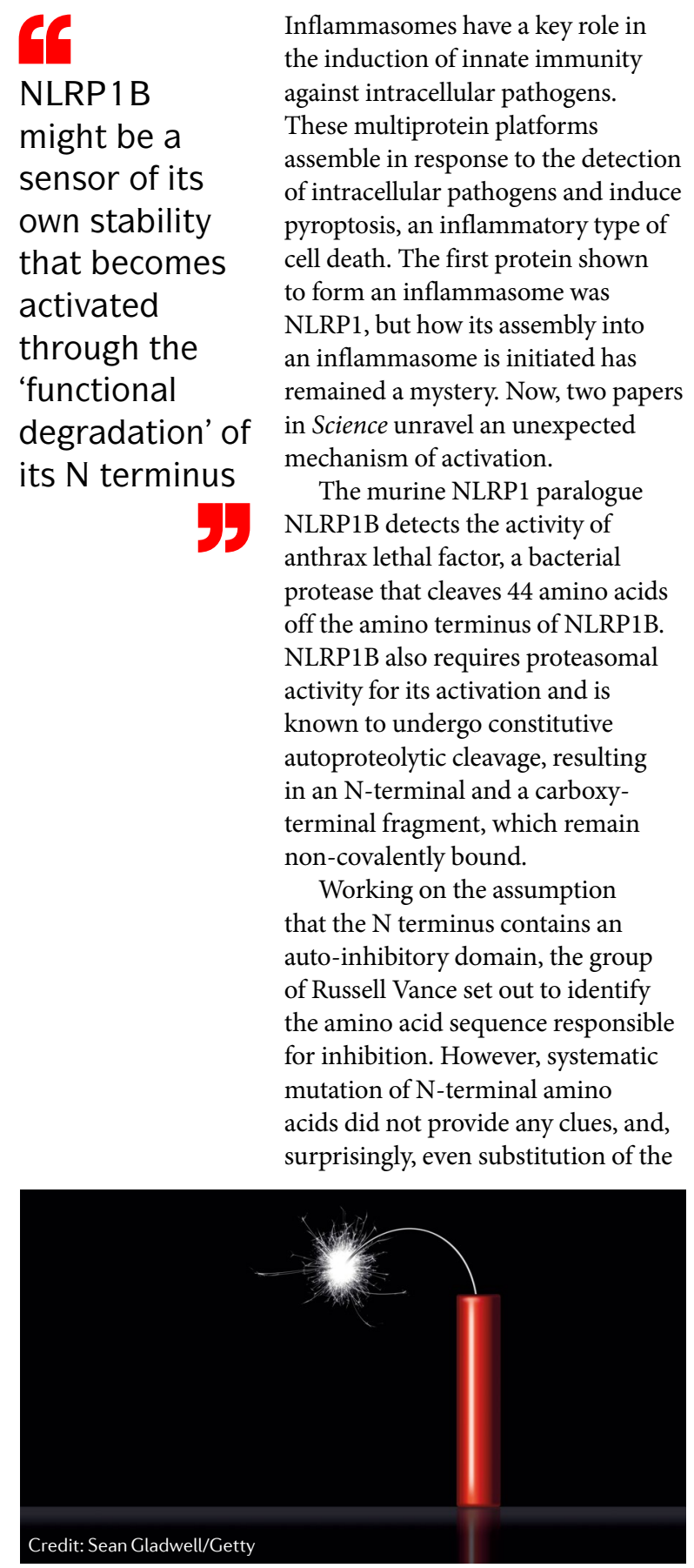

whole $\mathrm{N}$ terminus with an unrelated sequence did not alter the activity of the protein. They also found that cleavage of as few as ten amino acids from the $\mathrm{N}$ terminus of NLRP1B was sufficient to activate the protein. Moreover, enforced degradation of NLRP1B (by fusing a sequence to its $\mathrm{N}$ terminus that targets it for proteasomal degradation) induced inflammasome activation, even in the absence of lethal factor. This demonstrated that proteasomal degradation is not only necessary but also sufficient for activation.

These findings led the authors to conclude that NLRP1B might be a sensor of its own stability that becomes activated through the 'functional degradation' of its $\mathrm{N}$ terminus. This results in the liberation of the non-covalently bound bioactive C-terminal fragment, which self-assembles, recruits caspase 1 and thereby initiates pyroptosis. This, of course, raises the possibility that NLRP1B might sense any pathogen effector that induces proteasomal degradation of NLRP1B. Indeed, they found that the ubiquitin ligase Ipa7.8, secreted by Shigella flexneri, can also activate NLRP1B by ubiquitylating a site in its N-terminal fragment.

The second paper, by the group of Daniel Bachovchin, came to a similar conclusion, albeit through a different approach. Here, the authors used a genome-wide CRISPR-Cas9 knockout screen to identify genes that are required for NLRP1B-mediated pyroptosis. Cells were treated with either lethal factor or the DPP8/9 inhibitor Val-boroPro (VbP), which activates NLRP1B in a proteasome-dependent manner that does not involve cleavage of the $\mathrm{N}$ terminus. Several genes involved in the $\mathrm{N}$-end rule proteasomal degradation pathway, which is important for the degradation of proteins with destabilized N-terminal residues, were identified in the screen using lethal factor but not in the screen using VbP. Interestingly, inhibitors of the $\mathrm{N}$-end rule pathway had previously been reported to block lethal factor-mediated cell death.

Further biochemical analysis demonstrated that full-length NLRP1B is lost after treatment with either lethal factor or $\mathrm{VbP}$ but that considerable amounts of the $\mathrm{C}$-terminal fragment remain. The $\mathrm{N}$-terminal fragment was shown to be non-toxic, whereas the C-terminal fragment induced pyroptosis. Treatment with proteasome inhibitors prevented the targeted degradation of the $\mathrm{N}$-terminal fragment in response to lethal factor or VbP, and cells lacking $\mathrm{N}$-end rule proteins were protected from lethal factor-induced cell death but not from VbP-induced pyroptosis.

These results led the authors to conclude that the lethal factor-mediated N-terminal cleavage of NLRP1B leads to the activation of the $\mathrm{N}$-end rule pathway, targeting it for proteasomal degradation. Due to the autoproteolytic cleavage of NLRP1B, the C-terminal fragment is protected from this fate and subsequently seeds inflammasome assembly. In contrast, $\mathrm{VbP}$ appears to stimulate a different proteasomal degradation pathway.

Overall, these results suggest that NLRP1B may have evolved as a natural decoy that acts as a 'booby trap' for lethal factor and other pathogen-encoded activities. Insights into these mechanisms could form the conceptual basis for designing therapeutics targeted at NLRP1.

Alexandra Flemming

ORIGINAL ARTICLE Sandstrom, A. et al. Functional degradation: a mechanism of NLRP1 inflammasome activation by diverse pathogen enzymes. Science 364, eaau1330 (2019) | Chui, A. J. et al. N-terminal degradation activates the NLRP1B inflammasome. Science 364, 82-85 (2019) 